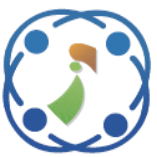

\title{
Solving Max-Min Separable Problem Using Hybrid Particle Swarm Optimization
}

\author{
Hanaa Khater ${ }^{1 *}$ \\ Ahmed ElSawy ${ }^{2}$ \\ Assem Tharwat ${ }^{3}$ \\ Ihab El-Khodary ${ }^{1}$ \\ ${ }^{1}$ Faculty of Computers and Artificial Intelligence, Operations Research and Decision Support Department, \\ Cairo University, Egypt \\ ${ }^{2}$ Faculty of Computers and Artificial Intelligence, Computer Science Department, Benha University, Egypt \\ ${ }^{3}$ College of Business Administration, The American University in the Emirates, Emirates \\ * Corresponding author's Email: hanaa_or@yahoo.com
}

\begin{abstract}
The max-min separable optimization problem is depended on max-min algebra. This optimization problem focuses on the worst case for minimization of objective function. Additionally, this problem has separable max-min constraints. It was solved in another article by using difficult and complicated algebraic method for finding one solution only. In this paper, the Max-Min Separable problem under Max-Min Separable Linear Constraints (MMSMMLC) is solved by hybrid PSO. The main idea of the presented algorithms is combining GA processes with PSO algorithm to be suitable for solving MMSMMLC optimization problem under all types of constraints (equality and/or inequality). Finally, the new algorithms applied through several benchmark functions show that the new algorithms present all optimal solutions in best result with $100 \%$ and improve the optimal solution in some cases rather than algebraic method.
\end{abstract}

Keywords: Hybrid-PSO, Max-min separable function, Max-Min linear constraint, Genetic algorithm (GA).

\section{Introduction}

The extremely algebra has many branches such as max, min denoted by $\vee, \wedge$ respectively, max-min and min-max ...etc. The two main operators max or min are usually followed by plus (+) or minus (-) operator. Many authors applied max or min operator followed by min or max operation. The merger between max-min or min-max shows the worst case of max-min or the best case of min-max optimization problems.

Cuninghame-Green introduced the main definition of max-plus algebra and min-max algebra. He introduced all its algebraic properties. He also calculated the exact period value for a given matrix [1]. The extremel algebra has a special case called max-separable function. The max-separable constraint was introduced as an optimization problem with unimodel function. This has been solved and implemented as an application in service-pointlocation problems [2]. Zimmermann solved the same disjunctive restrictions [3]. Tharwat and
Zimmermann proposed a method for solving optimization problems with a max-separable objective function and min-separable inequality constraints, and implemented that in location problems [4]. Moreover, Gavalec proposed a duality for max-separable problem of optimization, which would occur max-separable in both objective function and constraint without gap in duality [5]. Gavalec et. al presented algorithms for solving optimization problems under (max, min)-linear equations and/or inequality constraints and determined the feasible set of solution of each system of inequality constraint and system of equation [6].

Cimler et al. designed an algorithm for the unsolvability of (max, min) separable linear equation systems with coefficients in the unit interval (for fuzzy systems), or in an arbitrary real interval (general case). If the system has no solution, then the proposed algorithm computed the nearest vector to the right-hand side of the system for which the system is solvable [7].

A new definition of approximate solution was introduced by Xiao et al for the inconsistent system 
of max-min fuzzy relationship equations. They defined approximate solution that could minimize the equations biggest degree of dissatisfaction. They suggested a linear search algorithm on the basis of an auxiliary system with a parameter to find an approximate solution to an inconsistent max-min system [8].

In the last years, many scholars solved the maxplus or max-min function using heuristics searches and executed a comparison between PSO and genetic Algorithm for solving max-separable problem [9]. Other scholar applied max-algebra in location problem and using particle swarm algorithm for solving that problem [10]. Guo et al. solved constrained min-max optimization problems using constraint-activated differential evolution algorithm. They proved that a min-max algorithm could be used to solve a max-min problem without any algorithmic changes. Based on the theorems, they proposed a constraint-activated differential evolution to solve constrained min-max problems. The presented method consists of three components, propagation, constraint activation, and inner level evolution [11]. Khan and Rice suggested an alternative approach based on Max-Min algebra, where ternary logic functions are interpreted and realized using multiplecontrolled unary gates as Max-Min expressions. They also suggested realizations of several regulated unary gates on the quantum level. Using K-maps, they implemented minimization of Max-Min expressions and then suggested a system for minimizing and synthesizing ternary reversible circuits based on a hybrid genetic algorithm [12].

From the previous literature, it clear that the direction of modern methods for optimization is the hybrid algorithm. The concept of hybrid algorithm is mixing the two heuristic algorithms or meta-heuristic algorithm with another one to improve the performance of the algorithm reaching the optimal solution. Garg presented hybrid PSO_GA to solve optimization problem with constraint by mixing the operator of GA such as selection, crossover and mutation in population in PSO [13]. Also, Barroso et al. presented a hybrid PSO with mixed GA operator (mutation) for solving optimization of laminated composite structures [14]. Ahmed et al. solved a convex economic load dispatch (ELD) problem by a hybrid PSO-GSA which associated particle swarm optimization PSO and Gravitational Search Algorithm (GSA) to find global optimal solution of that problem [15]. Yan et. al presented a modern type of the hybrid particle swarm optimization (PSO) and Quasi-Newton (QN) algorithm on CPU-GPU platform using OpenCL to accelerate the training process of (artificial neural network_ANN) [16].
Other scholars presented a modern hybrid PSO with artificial Neural Network in Bankruptcy Prediction [17]. Mitras and Anwar applied hybrid PSO algorithm with modified conjugate gradient method for some image processing [18]. Xu et al. solved vehicle routing problem with time windows by applying hybrid PSO and GA by using one operator of GA as crossover to solve that problem by obtaining a more optimal result [19]. Niazy et al. solved the capacitated vehicle routing problem using hybrid chicken swarm optimization algorithm with Tabu Search [20]. Moreover, they solved the same problem using hybrid chicken swarm optimization with genetic algorithm [21].

In real situations, we resort to the worst case of (max or min) optimization to guarantee the optimality of objective function (target) and achieve the quality of optimizing the resources. Actually, if it can achieve the target in the worst case, it will achieve the target in other cases. So the ordinary use of that type of problem is mainly in economic application or to control the resources such as the capacity of roads, electricity networks, water networks ...etc.

From the previous literature we find that all researches didn't solve the max-min separable optimization problem with meta-heuristics algorithm. It solved only max-plus separable function $[14,17]$.

The previous work focuses on using algebraic method and not using the meta-heuristic algorithms to benefit from the power of those methods for finding the optimal solution without complexity computational.

The aim of this paper is to solve (MMSMMLC) problem by using meta heuristic Algorithms to find the optimal solution specially the (MMSMMLC) problem which is NP-hard [3]. So, the new algorithms solve (MMSMMLC) problem without converting the equality constraint into (two inequality constraint ( $\leq$ and $\geq$ ) as the algebraic method [22]. This paper shows the abilities of metaheuristics algorithm to achieve the optimal solution of each separable objective functions and finally compute the maximum of all.

This paper is organized as follows :- Section 2: presents the theoretical background about the MaxMin-separable optimization problem under max-min constraints and presents the algebraic method for solving that problem depending on lemmas were presented by Gavalec et.al [6] . Section 3: presents back ground about uses algorithm such as Partial Swarm Optimization algorithm and genetic algorithm. Section 4: presents an algorithm for determine maximum point in feasible set of solution for the max-min separable problem. Section 5 presents a hybrid PSO with genetic Algorithm for solving the 
presented problem. Section 6: tests the modified algorithms through numerical examples and presents the result. Section 7: presents the conclusion from the results and suggests the future work to be presented.

\section{Theoretical background}

Gavalec et al. presented the general form of an optimization problem with max-min separable objective function subject to max-min linear equality/inequality constraints, and solved that problem by algebraic method with real number. Actually, it is not easy to solve the max-min separable objective variable under max-min constraints because max-min separable constraints must be satisfied by vector of decision variables.

The algebraic method solved that problem with two separated algorithms. One, for solving that problem under inequality constraints (greater than or equal and less than or equal) in [22], and another for solving the same optimization problem under equality constraints only in [6]. The algebraic method for solving the presented optimization problem under mix constraint between equality and inequality constraint must be converted equality constraint into double inequality constraint with type greater than or equal and less than or equal.

\subsection{Max-Min separable objective function under max-min linear constraint (MMSMMLC)}

optimization problem with mixed (inequality and/or equality) constraint a following:

$F(X)=$

$\max \left(\min \left(f_{1}\left(x_{1}\right), \min \left(f_{2}\left(x_{2}\right), \ldots, \min \left(f_{\text {nvar }}\left(x_{n v a r}\right)\right)\right.\right.\right.$

\section{Subject to:}

$$
\begin{aligned}
& \max \left(a_{i j} \wedge x_{j}\right)=b_{i}, \quad i \in I 1 \\
& \max \left(a_{i j} \wedge x_{j}\right) \leq b_{i}, \quad i \in I 2 \\
& \max \left(a_{i j} \wedge x_{j}\right) \geq b_{i}, \quad i \in I 3 \\
& l x_{j} \leq x_{j} \leq u x_{j}
\end{aligned}
$$

Where $f_{j}: R^{\text {nvar }} \rightarrow R^{\text {nvar }}$ are continuous functions, $x_{j} \in \boldsymbol{M}$ are decision variables, $\boldsymbol{M}$ is a feasible set of solution, $\quad I 1=\{1,2, \ldots, k\}, I 2=\{k+$ $1, \ldots, m\}, I 3=\{m+1, \ldots, n\} I=I 1 \cup I 2 \cup I 3, I 1$ for $=$ constraints and $I 2$ for $\leq$ constraints,$I 3$ for $\geq$ constraints, $j=\{1,2, \ldots, n v a r\}, I=\{1,2, \ldots, n\}$, $x_{j}=\left(x_{1}, x_{2}, \ldots, x_{n v a r}\right) \in R, a_{i j} \in R, b_{i} \in R \forall i \in$ $I, j \in J$.

\subsection{Algebraic Method.}

The algebraic method was investigated by [22] solved the (MMSMMLC) optimization problem without equality constraints. The algorithm depends on determining the feasible set of solution of each decision variable by closed interval using the following Lemma 1 and Lemma 2 after that select the minimum of objective function and set that as optimal solution. That algorithm is manipulating an inequality constraint to find one optimal solution only even if the objective function has multi-optimal solutions. The algebraic method is valid in case of linear objective and uni-modal nonlinear objective function, but in case of multimodal nonlinear objective function all possible optimal solution can't be founded. Moreover, it can't solve problem which has inequality constraint and equality constraint in the same problem without converting each equality constraint into twice inequality constraints. On the other hand, the same authors investigated an algebraic algorithm for solving that type of problem under equality constraint only and started search for optimal solution with feasible point which it was determined by the following lemmas 3 and 4

The following four lemmas are presented and proved by Gavalec et al [6] in details.

Lemma 1:

$$
\begin{aligned}
V_{i j}^{\leq}=\left\{x_{j}\right. & ;\left(a_{i j} \wedge x_{j}\right) \leq b_{i} \\
& \text { and } \left.l x_{j} \leq x_{j} \leq u x_{j}\right\}
\end{aligned}
$$

Therefore $V_{i j}^{\leq}$is the feasible set of the system and its upper bound represents the maximum element for the feasible set M satisfying Eq. (3) and Eq. (5).

For arbitrary $i$ and $j, V_{i j}^{\leq}$can be reformulated as follows:

$$
\begin{gathered}
V_{i j}^{\leq} \\
=\left\{\begin{array}{cc}
{\left[l x_{j}, u x_{j}\right]} & \text { if } a_{i j} \leq b_{i} \\
{\left[l x_{j}, u x_{j} \wedge b_{i}\right]} & \text { if } a_{i j}>b_{i} \text { and } b_{i} \geq l x_{j} \\
\varnothing & \text { if } a_{i j}>b_{i} \text { and } b_{i}<l x_{j}
\end{array}\right.
\end{gathered}
$$

Since $x_{j} \in \boldsymbol{M} \Rightarrow x_{j} \in \bigcap_{i \in I_{2}} V_{i j}^{\leq} \forall j \in J$,

Therefore $V_{i j}^{\leq}=\varnothing \Leftrightarrow \boldsymbol{M}=\emptyset$, 
So, $u x_{i j}$ can be defined as an upper bound of $\mathrm{V}_{\mathrm{ij}}^{\leq} \neq \varnothing$ as a following:

$$
u x_{i j}=\left\{\begin{array}{lc}
u x_{j} & a_{i j} \leq b_{i} \\
u x_{j} \wedge b_{i} & a_{i j}>b_{i} \text { and } b_{i} \geq l x_{j}
\end{array}\right.
$$

Therefore the upper bound $u x_{j}^{\max }=\min _{i \in I 2} u x_{i j}$ the element $u x_{j}^{\max }$ is the maximal element of feasible set of solution $\boldsymbol{M}$ satisfying Eq.(3) and Eq. (5) and replacing by new upper bound $u x_{j}^{\max }$

Remark: For any $x_{j} \in\left[l x_{j}, u x_{j}\right]$ if $x_{j} \leq u x_{j}^{\max }$ we will have to find the solution for the system of Eq. (2) and Eq.(4) otherwise there is no solution for MMSMMLC problem.

\section{Lemma 2:}

The following lemma determines the set of feasible solution for greater than or equal constraint Eq. (4) with regard of satisfying the system of equation Eq. (3) and Eq. (5) by defining $\mathrm{V}_{\mathrm{ij}}^{\geq}$as follows:

$$
\begin{aligned}
& V_{i j}^{\geq}=\left\{x_{j}:\left(a_{i j} \wedge x_{j}\right) \geq b_{i} \& l x_{j} \leq x_{j} \leq\right. \\
& \left.\boldsymbol{u} \boldsymbol{x}_{\boldsymbol{j}}\right\}
\end{aligned}
$$

Therefore $\boldsymbol{V}_{\boldsymbol{i} \boldsymbol{j}}^{\geq}$is the feasible set of the system and its upper bound represents the maximum element for the feasible set $\boldsymbol{M}$ satisfying (2) and (4)

For arbitrary $i$ and $j, V_{i j}^{\geq}$can be reformulated as follows:

$$
\begin{aligned}
& V_{i j}^{\geq} \\
& =\left\{\begin{array}{cl}
{\left[b_{i} \vee l x_{j}, u x_{j}\right]} & \text { if } a_{i j} \geq b_{i} \& b_{i} \leq u x_{j} \\
\varnothing & \text { if } a_{i j}<b_{i} \text { or } b_{i}>u x_{j}
\end{array}\right.
\end{aligned}
$$

Where $x_{j} \in M=\bigcap_{i \in I 3} \bigcup_{j \in J} V_{i j}^{\geq}, \forall j \in J$

Therefore $V_{i j}^{\geq}=\emptyset \Leftrightarrow M=\emptyset$

The following lemmas (3) and (4) present the properties of set $\boldsymbol{M}$ satisfying Eq.(2) and Eq.(5)

Let us define:

$$
I_{j}=\left\{i \in I 1 \text { and } a_{i j}>b_{i}\right\}
$$

Which represent the position of constraint $i$ for any fixed $j \in J$ satisfying $a_{i j}>b_{i}$;

$S_{j}\left(x_{j}\right)=\left\{k \in I 1 ; a_{k j} \wedge x_{j}=b_{k}\right.$

Which represent the position of constraint $\mathrm{i}$ for any fixed $j \in J$ satisfying $a_{k j} \wedge x_{j}=b_{k}$;

$$
M=\left\{x_{j} \in M \text { and } x \leq u x_{j}\right\}
$$

Lemma 3:

$$
\begin{gathered}
V_{i j}=\left\{x_{j} ;\left(a_{i j} \wedge x_{j}\right)=b_{i} \text { and } l x_{j} \leq x_{j}\right. \\
\left.\leq u x_{j}\right\}
\end{gathered}
$$

For arbitrary $i$ and $j, V_{i j}$ can be reformulated as follows:

$$
V_{i j}=\left\{\begin{array}{c}
\left\{b_{i}\right\} \quad \text { if } a_{i j}>b_{i} \text { and } b_{i} \leq u x_{j} \\
{\left[b_{i}, u x_{j}\right] \text { if } a_{i j}=b_{i} \text { and } b_{i} \leq u x_{j}} \\
\varnothing \quad \text { if } a_{i j}<b_{i} \text { and } b_{i}>u x_{j}
\end{array}\right.
$$

$V_{i j}^{\bar{j}}$ represents the new feasible set of solution including the optimal solution.

Lemma 4:

Set for all $i \in I 1$ and $j \in J$ which $x_{j}^{(i)}$ is a maximum element in $\boldsymbol{M}$ for arbitrary $i$

$$
\begin{aligned}
& x_{j}^{(i)}= \\
& \left\{\begin{array}{l}
b_{i} \quad \text { if } a_{i j}>b_{i} \text { and } b_{i} \leq u x_{j} \\
u x_{j} \quad \text { if } a_{i j}=b_{i} \text { and } b_{i} \leq u x_{j} \text { or } V_{i j}=\varnothing
\end{array}\right. \\
& I_{j}=\left\{i \in I 1 \text { and } a_{i j} \geq b_{i}\right\} \forall j \in J
\end{aligned}
$$

And let $\hat{\mathrm{x}}_{\mathrm{j}}$ is a maximum element in $\boldsymbol{M}$

$$
\hat{x}_{j}=\left\{\begin{array}{cl}
\min _{k \in I_{j}} x_{j}^{(k)} & \text { if } I_{j} \neq \varnothing \\
u x_{j} & \text { if } I_{j}=\varnothing
\end{array}\right.
$$

Let $S_{j}\left(\hat{x}_{j}\right)=\left\{k \in I 1 ; x_{j}^{(i)}=\hat{x}_{j}\right\} \forall j \in J$

And the following statements hold:

$$
\hat{x}_{j} \in M \Leftrightarrow x_{j} \in \cup_{J \in j} S_{j}\left(\hat{x}_{j}\right)=I 1
$$

If $\boldsymbol{M} \neq \varnothing$, then $\boldsymbol{M}$ and $\forall x \in \boldsymbol{M} \Leftrightarrow x \leq \hat{x}$,

So $\hat{x}$ is maximum element in $M$

\section{Background about PSO and GA algorithms}

Particle swarm optimization (PSO) was investigated in 1995 as a stochastic technique of global search. PSO is a method inspired by the behaviour of birds. The following sub-sections presents some types of meta-heuristics algorithm which will be used in this paper such as Particle Swarm Optimization algorithm (PSO) and Genetic Algorithm (GA). These algorithms will be modified to solve the MMSMMLC. Moreover, this paper will 
present a hybrid particle swarm optimization and genetic algorithm to solve MMSMMLC problem.

\subsection{Particle swarm optimization (PSO)}

The PSO algorithm is stimulated from swarm performance such as bird flocking. PSO has been widely used in optimization problem as a type of meta-heuristics algorithm for solving NP-hard problem. The parameters used in PSO algorithm are defined in Table 1 to explain the idea of PSO algorithm.

The PSO Algorithm consists of npop particle $i \in$ npop fly around dimension to search in feasible region to find the optimal solution. Initialize the position of each particle $x_{i} \in\left[x_{\min }, x_{\max }\right]$ and $v_{i} \in$ $\left[v_{\text {min }}, v_{\text {max }}\right]$ randomly. The velocity $v_{i}$ and particles $x_{i}$ must be updated according to the following Eq. (14) and Eq. (15) in each iteration.

$$
\begin{aligned}
v_{i}(t+1)=w & \cdot v_{i}(t) \\
& +c_{1} \cdot r_{1}\left(x_{i}^{\text {best }}(t)\right. \\
& \left.-x_{i}(t)\right) \\
& +c_{2} \cdot r_{2}\left(x_{i}^{\text {global }}(t)\right. \\
& \left.-x_{i}(t)\right) \\
x_{i}(t+1) & =x_{i}(t)+v_{i}(t+1)
\end{aligned}
$$

Where $r_{1}$ and $r_{2}$ are uniform random variable in range $[0,1], c_{1}$ and $c_{2}$ are constant which was known as accelerations coefficient and $\mathrm{w}$ was known as inertia weight within range $[0,1]$. Inertia weight was affecting the type of solution. If inertia weight is small, it will fall on local optimal. Also if inertia weight is large, the global optimal will be found.

After each iteration select the best particle of each swarm $x_{i}^{\text {best }}$ according to the nature of problem

\begin{tabular}{|c|c|}
\hline \multicolumn{2}{|r|}{ Algorithms } \\
\hline$I 1$ & Number of equality $=$ constraints \\
\hline$I 2$ & $\begin{array}{l}\text { Number of less than or equal } \leq \\
\text { constraints }\end{array}$ \\
\hline$I 3$ & $\begin{array}{l}\text { Number of greater than or equal } \\
\geq \text { constraints }\end{array}$ \\
\hline$a_{i j}$ & Right hand side variable \\
\hline$b_{i}$ & Left hand side variable \\
\hline$x_{j} \in\left[l x_{j}, u x_{j}\right]$ & $\begin{array}{l}\text { Decision variable } x_{j} \text { must be } \\
\text { between lower bound } l x_{j} \text { and } \\
\text { upper bound } u x_{j}\end{array}$ \\
\hline$v_{j} \in\left[l v_{j}, u v_{j}\right]$ & $\begin{array}{l}\text { Velocity } v_{j} \text { of particle must be } \\
\text { between lower bound } l v_{j} \text { and } \\
\text { upper bound } u v_{j}\end{array}$ \\
\hline$t_{\max }$ & Maximum number of iterations \\
\hline npop & Number of particle \\
\hline $\begin{array}{l}\text { w } \\
\in[\text { wmin,wmax }]\end{array}$ & $\begin{array}{l}\text { Inertia weight } w \text { must be between } \\
\text { minimum weight wmin and } \\
\text { maximum weight wmax }\end{array}$ \\
\hline $\mathrm{PC}$ rate & Crossover rate, $P C \in[0,1]$ \\
\hline PM rate & Mutation rate, $P M \in[0,1]$ \\
\hline$x_{i}^{b e s t}$ & $\begin{array}{l}\text { In each iteration select the best } \\
\text { particle of each swarm } x_{i}^{\text {best }} \\
\text { according to natural of problem } \\
\text { (Max or Min) and after each } \\
\text { iteration select }\end{array}$ \\
\hline$x_{i}^{\text {global }}$ & $\begin{array}{l}\text { global best particle } x_{i}^{\text {global }} \text { for all } \\
\text { iterations }\end{array}$ \\
\hline$r_{1}$ and $r_{2}$ & $\begin{array}{l}\text { are uniform random variables in } \\
\text { range }[0,1]\end{array}$ \\
\hline$c_{1}$ and $c_{2}$ & are constant \\
\hline
\end{tabular}
(Max or Min) and after each iteration select the global

\begin{tabular}{l} 
Algorithm $1:$ pseudo code Particle Swarm \\
Optimization \\
\hline Initialize randomly position and velocity of all particles. \\
Do \\
For $t$ to $t_{\text {max }}$ \\
For $i$ to npop \\
$\quad$ Set $x_{i}^{\text {best }}$ and $x_{i}^{\text {global }}$ \\
Calculate particle velocity according to Eq. \\
Evaluation the objective function value (fitness value) \\
End \\
End \\
While A satisfactory optimal solution has been found \\
\hline
\end{tabular}

Table 1. Parameters that using in different PSO

best particle $x_{i}^{\text {global }}$. The benefits of two variable $x_{i}^{\text {best }}$ and $x_{i}^{\text {global }}$ are played as memory of algorithm to improve the solution in the next iteration till reaching the optimal solution. Algorithm 1 is briefing the pseudo code of Particle swarm Optimization (PSO) Algorithm.

\subsection{Genetic algorithm}

The genetic algorithm (GA) is an important stochastic search algorithm used in the last century to solve optimization problems. Different problems such as control problem, image processing, and route planning at construction sites have been commonly and effectively implemented [23]. GA varies in the following characteristics from most traditional calculus-based search algorithms: no constraint on the continuity or discreteness of the search space, parallel population computation. The idea of GA comes from the Evolutionary Algorithms (EA) family. So, it produces solutions through the use of 
natural influenced evolution techniques in optimization problems [24].

Genetic algorithm starts with initializing the offspring randomly then, for each iteration executing several processes such as selection process which selects the good parents according to selection method, crossover process and mutation process. All of those processes are executed by different strategies and then the result of these processes tested fitness of objective function. Finally, select the accepted solution according to objective function (maximize or minimize) and repeating the previous steps till reaching the maximum number of iterations. The following pseudo code explains the (GA) algorithm, see Algorithm 2

\begin{tabular}{l}
\hline Algorithm 2: pseudo code Genetic Algorithm \\
\hline Initialize offspring \\
Do \\
For $t \leq t_{\max }$ \\
$\quad$ Selection \\
Crossover \\
Mutation \\
Test fitness of objective function \\
Accepted and produced new offspring \\
End \\
While A satisfactory optimal solution has been found \\
\hline
\end{tabular}

\section{Proposed algorithms.}

The MMSMMLC problem will be solved by using algebraic method which is described in section (2.1) then we will present Max-Point Algorithm to determine the feasible set of solution for all types of constraints greater than or equal, less than or equal and equality constraints. This algorithm helps us to solve MMSMMLC problem by new Hybrid-PSO with genetic algorithms with (crossover and mutation operation or both) which are called MMS-PSO-Cross, MMS-PSO-Mut and MMS-PSO-GA for solving max-min separable optimization under max-min linear constraints which will be listed in the next section. Moreover modify PSO algorithm and LAPSO algorithm to be suitable for solving MMSMMLC problem.

\section{Max-point algorithm (MPA)}

The idea of this algorithm (MPA) depends on the previous Lemma 1 and Lemma 3 and Lemma 4. That algorithm split all constraint into three parts I1 for equality constraint, I2 for less than or equal constraint and $I 3$ for greater than or equal constraint. So, the following algorithm starts with $I 2$ constraint and determines maximum point of the feasible region according to Lemma (1) then from the produced new maximum point tested of satisfaction for all constraint $I 1$ by decreasing that maximum point to produce feasible region for $I 1$ and $I 2$ constraint. Finally, examine that new maximum point for satisfying of $I 3$ else if the new maximum point doesn't satisfy all constraints then the set of feasible solution $\mathrm{M}$ is empty, in the other words if $a_{i j}<$ $b_{i}$ or $b_{i}>u x_{j}$ then $\mathrm{M}=\emptyset$ for all $i \in I 3$ and $j \in$ nvar according to Lemma 2.

Note, The following Algorithm obtains maximum point in a set of feasible solution $\mathrm{M}$ for decision variables $x_{j}$. That algorithm whose efficient in case of equality constraints and less than or equal $(\leq)$ constraints but that is not effective in case of greater than or equal $(\geq)$ constraints to change the upper limit of decision variable because the nature of stochastic search can reach optimal solution without need to start search with feasible point. The Max-PointAlgorithm is explained in details in the following steps and Algorithm 3 for its pseudo code.

Step 1: Define $I 1, I 2, I 3, a_{i j}, b_{i}, j \in$ nvar $l x_{j}, u x_{j}$ and $i \in I 1, I 2$ and $I 3$

Step 2: Start with less than or equal constraints and determine the maximum element of feasible set of solution which satisfies all less than or equal constraints defined as $X \max$, for all $i \in I 2$ and $j \in$ nvar then set $x u=X \max$ according to Lemma 1 , if $V_{i j}^{\leq}=\emptyset$ for any $i \in$ $I 2$ and $\mathrm{j} \in$ nvar then $\mathrm{M}=\emptyset$. So, there is no feasible solution, Stop.

Step 3: Determine the maximum point $u x$ (new) of equality constraints according to Lemma 3 and Lemma 4 and set $u x=u x($ new) then check if $a_{i j}>b_{i}$ and $b_{i}<l x_{j}$ for all $i \in I 2$ and $j \in$ nvar then $\mathrm{M}=\varnothing$. So, there is no feasible solution, Stop.

Step 4: Check the feasibility of new maximum point $x u$ in greater than or equal constraints by checking if $a_{i j}<b_{i}$ or $b_{i}>u x_{j}$ for all $i \in$ $I 3$ and $j \in$ nvar then $\mathrm{M}=\varnothing$ so there is no feasible solution, Stop. Otherwise, return $u x$ Stop.

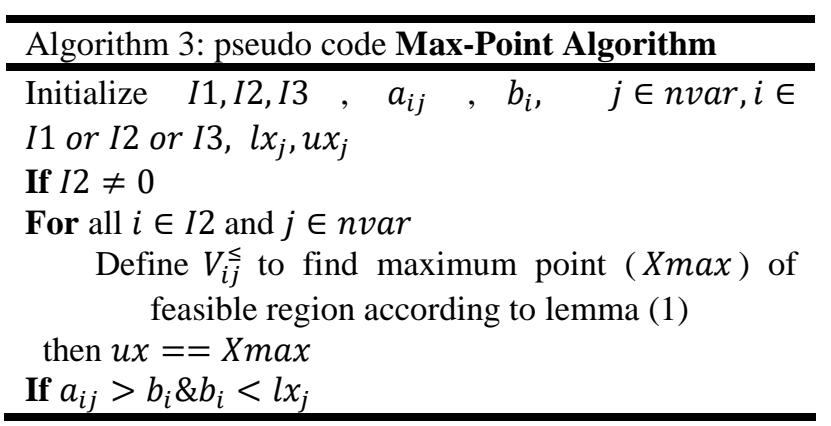




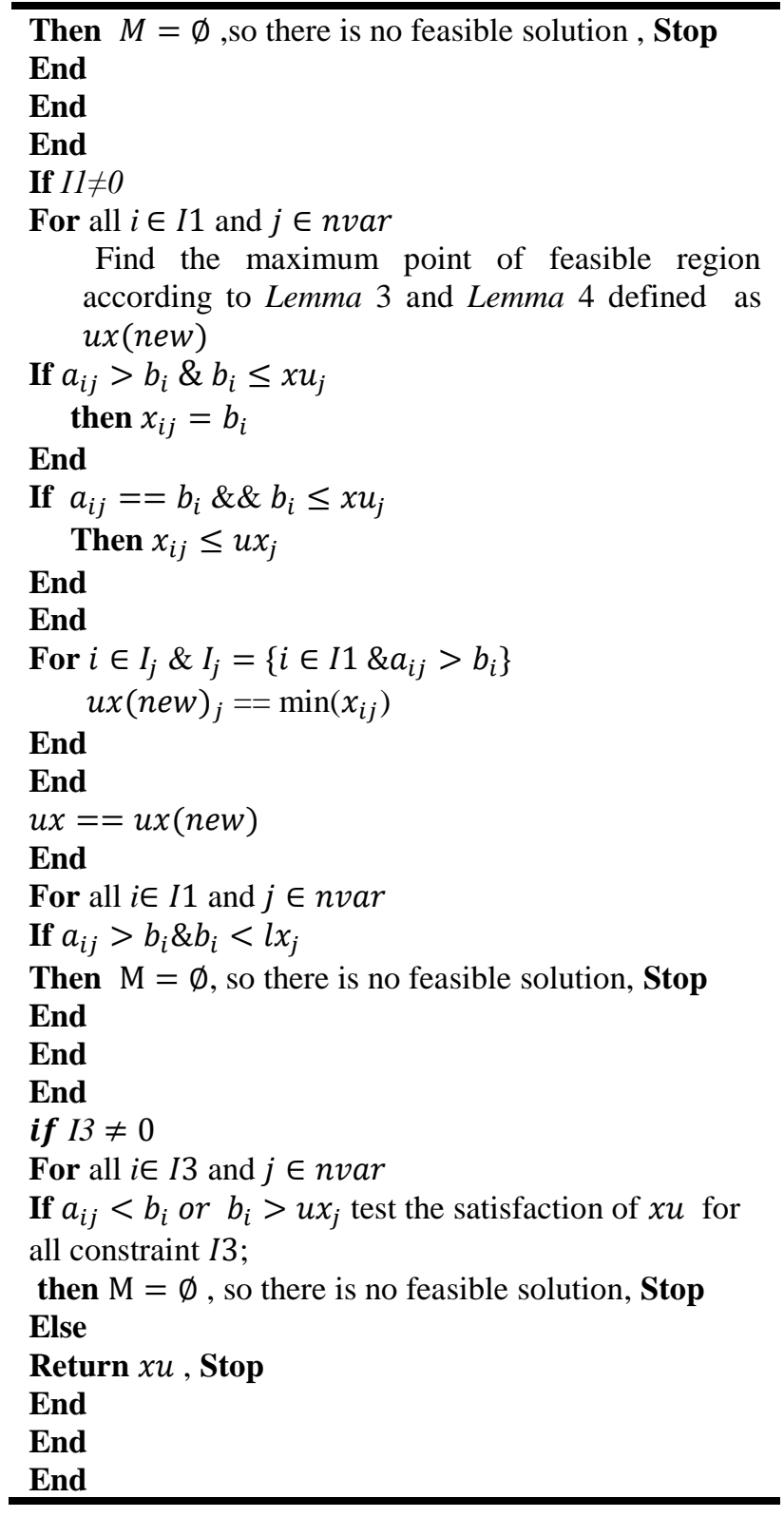

\section{Max-min separable PSO-GA algorithm (MNS-PSO-GA)}

The MMSMMLC problem was solved by two separated algorithms. The first algorithm which solved that problem with only inequality constraints [22] and the second algorithm which solved the optimization problem including equality constraints only using algebraic method [6]. The proposal algorithm solved MMSMMLC problem with all types of constraint $(\leq, \geq$ and $=$ ) by using hybrid particle swarm optimization with genetic algorithm as a stochastic search of all optimal solutions.

Actually, any type of PSO algorithms can't solve the MMSMMLC optimization problem without modification for many reasons: the first reason is PSO was designed as an algorithm optimization problem without any constraints and treated this problem by a penalty function [25] to guarantee satisfying all constraints. The second reason is the max-min separable constraint in case of equality constraint can't be suitable without transferring equality constraint into pair of inequality constraints. The third reason is the nature of max-min separable objective function which requires finding the optimal of the separated objective functions $f_{j}\left(x_{j}\right)$. Finally, find the maximum of all separable functions. So, the PSO optimizes only $F(x)$ and neglected optimizing each separating objective function $f_{j}\left(x_{j}\right)$ included in $F(x)$ due to the previous reasons the next modified algorithms to optimize separates $f_{j}\left(x_{j}\right)$.

The proposed algorithm finds the optimal solution of MMSMMLC problem without transforming the equality constraint into pair of inequality constraints as in algebraic method. The MNS-PSO-GA Algorithm is considered as hybridPSO which mixed PSO with some process of GA to achieve the benefits of PSO and GA for solving optimization problem. The MNS-PSO-GA Algorithm will start with determining the set of feasible solution by applying Max-point-Algorithm (MPA) to decrease the chance of search outside the feasible region. So, it doesn't need penalty function because the search of solution applies within feasible region, then determine the dynamic weight Eq. (22) recommended by literature [26]. Next, it implements PSO algorithm for each population and executes crossover process and mutation process. Finally, test the fitness of objective function to find optimal solution. The following steps explain the next steps in details.

Step 1: This algorithm starts by entering the parameter of PSO Algorithm, $t_{\max }$, $w_{\text {max }}, w_{\text {min }}, c 1, c 2, r 1, r 2$

Step 2: Enter the parameters of constraints $a_{i j}, b_{i}$ where $\quad i \in I, d \in$ nvar, $l x_{i}, u x_{i} \quad$ and objective function $F(X)=$ $\max \left(\min \left(f_{1}\left(x_{1}\right), \min f_{2}\left(x_{2}\right), \ldots, \operatorname{minf}_{d}\left(x_{d}\right)\right.\right.$.

Step 3: Apply Algorithm Max-Point-Algorithm to determine the new upper limit of decision variable according to feasible set of solution, see Algorithm (3).

Step 4: Initialize randomly the decision variable $x_{i} \in$ $\left[l x_{i}, u x_{i}\right], v_{i} \in\left[l v_{i}, u v_{i}\right]$, For all $i \in$ npop.

Step 5: Set $x_{i}^{b e s t}$ for each particle swarm, Set $x^{\text {global }}=u x$ for first iteration to guarantee start search with feasible point especially in case of equality constraints.

Step 6: Apply the crossover process in particle position hild $1\left(x_{t}\right), \operatorname{Pchild} 2\left(x_{t}\right)$ and velocity as a following 


$$
\begin{aligned}
& P \text { child } 1\left(x_{t}\right)=P C \times P \operatorname{Parent} 1\left(x_{t}\right)+ \\
& (1-P C) \times P \text { Parent } 2\left(x_{t}\right) \\
& P \text { child } 2\left(x_{t}\right)=P C \times P \operatorname{Parent} 2\left(x_{t}\right)+ \\
& (1-P C) \times P \operatorname{Parent} 1\left(x_{t}\right)
\end{aligned}
$$

Which $\mathrm{PC}$ rate of crossover $P C \in[0,1]$, update velocity $\operatorname{Pchild} 1\left(v_{t}\right), \operatorname{Pchild} 2\left(v_{t}\right)$

$$
\begin{aligned}
& \operatorname{Pchild} 1\left(v_{t}\right)= \\
& \frac{\operatorname{PParent} 1\left(v_{t}\right)+P \operatorname{Parent} 2\left(v_{t}\right)}{\left|\operatorname{PParent} 1\left(v_{t}\right)+P \operatorname{Parent} 2\left(v_{t}\right)\right|}\left|\operatorname{PParent} 1\left(v_{t}\right)\right|
\end{aligned}
$$

$$
\begin{aligned}
& \operatorname{Pchild} 2\left(v_{t}\right)= \\
& \frac{\operatorname{PParent} 1\left(v_{t}\right)+P \operatorname{Parent} 2\left(v_{t}\right)}{\left|\operatorname{PParent} 1\left(v_{t}\right)+P \operatorname{PParent} 2\left(v_{t}\right)\right|} \mid \text { PParent } 2\left(v_{t}\right) \mid
\end{aligned}
$$

Step 7: Apply the mutation process with given mutation rate and update velocity $v_{i}$ and position $x_{i}$ of particle $x_{i}$ for all npop as following equations:

$$
\begin{aligned}
& x_{i}=M u \times\left(u x_{i}-l x_{i}\right)+l x_{i} \\
& v_{i}=M u \times\left(u v_{i}-l v_{i}\right)+l v_{i}
\end{aligned}
$$

$M u$ is a uniform random variable $[0,1]$

Step 8: Calculate dynamic weight $\mathrm{w}$ is weight linear decreasing function which was presented [26] and proved the efficiency of that weight to find solution.

$$
w=\left(w_{\max }-w_{\min }\right) \times \frac{t_{\max }-t}{t_{\max }}+w_{\min }
$$

for all $t_{\max }$

Step 9: update $v_{i}$ and $x_{i}$ according Eq. (14) and Es.

(15) for all $i \in$ npop

Find the optimal value of each separable objective function separately

Step 10: Evaluate fitness of separable objective function for $i \in$ npop

$F\left(X_{i}\right)=\left(\operatorname{minf}_{1}\left(x_{i 1}\right), \operatorname{minf}_{2}\left(x_{i 2}\right), \ldots, \operatorname{minf}_{d}\left(x_{i d}\right)\right.$, SwapBest $=x_{i}$

Step 11: Update $x_{i}^{\text {best }} \forall d \in$ nvar which nvar is a dimension of decision variable

If $\quad f_{d}\left(x_{i d}\right)<=f_{d}\left(x_{i d}^{b e s t}\right)$;

then $\quad$ SwapBest ${ }^{(d)}=x_{i d}$; End

If SwapBest satisfy all constraint,

then $x_{i}^{\text {best }}=$ SwapBest; End

Set SwapGlobal $=x_{i}^{\text {global }}$

Step 12: Update $x_{i}^{\text {global }} \forall d \in$ nvar which nvar is a dimension of decision variable

If $f_{d}\left(x_{i d}^{\text {best }}\right)<=f_{d}\left(x_{i}^{\text {global }}\right)$, then SwapGlobal ${ }^{(d)}=x_{i d}^{\text {best }} ;$ End

If SwapGlobal satisfy all constraint, then $x_{i}^{\text {global }}=$ SwapGlobal; End

Repeat Step 6 to Step 12 till to end number of maximum iteration $\boldsymbol{t}_{\text {max }}$

Step 13: maximum of $F\left(X_{i}^{\text {global }}\right)$ is objective function with optimal solution $x_{i}^{\text {global }}$. Stop

\section{Algorithm 4: Pseudo code Max-Min Separable optimization problem with max-min separable constraint Hybrid-PSO-GA Algorithm (MNS-} PSO-GA)

Read parameter of algorithm
$n p o p, t_{\max }, w_{\max }, w_{\min }, c 1, c 2, r 1, r 2$

Read parameter of optimization problem $a_{i j}, b_{i}$ where $I \in I 1 \cup I 2 \cup I 3$, nvar and objective function

$\mathrm{F}(\mathrm{X})$

$=\max \left(\min _{1}\left(x_{1}\right), \operatorname{minf}_{2}\left(x_{2}\right), \ldots, \min f_{n v a r}\left(x_{n v a r}\right)\right.$

$l x=\left\{l x_{1}, l x_{2}, \ldots, l x_{n v a r}\right\}, u x=$ $\left\{u x_{1}, u x_{2}, \ldots, u x_{n v a r}\right\}$.

Determine maximum point of set of solution and set as $u x$ by applying(MPA) algorithm

Initialize randomly position $x_{0}$ and velocity $v_{0}$ of all particles.

Do

For $t \leq t_{\max }$

Applying crossover process in position according to Eq. (16) and Eq. (17) and velocity according to Eq. (18) and Eq.

And/Or

Applying mutation process in position according to Eq. (20)and velocity according to Eq. (21)

Set weight $w$ according Eq. (22)

For $i \leq n p o p$

Set $x_{i}^{\text {best }}$, and $x_{i}^{\text {global }}$

Evaluation the objective function value (fitness value)

Set SwapGlobal $=x_{i}^{\text {global }}$

For $\mathrm{d}=1:$ nvar

$$
\begin{aligned}
& \text { If } \operatorname{minf}_{1}\left(x_{(i) d}\right) \leq \operatorname{minf}_{1}\left(x_{(i) d}^{\text {best }}\right) \\
& \quad \operatorname{SwapGlobal}(\mathrm{d})=x_{(i) d}
\end{aligned}
$$

If SwapGlobal satisfy all constraint $I$

Then

$$
\text { End }
$$$$
x_{i}^{\text {global }}=\text { SwapGlobal }
$$

\section{End}

\section{End}

End

End 
While A satisfactory optimal solution $x_{i}^{\text {global }}$ has been found

Calculate maximum of $\mathrm{F}\left(x^{\text {global }}\right)$, Stop.

The processes of genetic algorithm (crossover and mutation) are applied into three Hybrid-PSO algorithms, the first algorithm applies only crossover process which is called MNS-PSO-GA-Cross and the second algorithm applies only mutation process which is called MNS-PSO-GA-Mut and the third algorithm applies two processes (crossovermutation) which are presented in the previous algorithm called MNS-PSO-GA

Similarly, in PSO algorithm can be modified to be suitable for solving MMSMMLC by applying the Max-Point Algorithm and continuing the steps of PSO algorithm, then testing the fitness of objective function to reach optimal solution which can be called as MNS-PSO. On the other hand, LOAPSO algorithm [27] is a type of PSO which can be modified to solve MMSMMLC in the same way and called as MNS-LOAPSO. In this paper, all presented algorithms are compared with algebraic method [22] and will observe the efficiency of the proposal algorithms for solving MMSMMLC problem through numerical examples.

\section{Numerical examples and results}

This section presents benchmark P1 to P5 were presented in [22] and the new numerical examples P6 to P10 present to test the proposal algorithms. The benchmark functions and numerical examples present all types of optimization problem to test the proposal algorithms such as multimodal objective function in P3, P7, P9 and P10. Moreover, they presented all types of constraints such as equality constraints only through P1 to P3, mixed constraints (equality and inequality in the same problem) through $\mathrm{P} 4, \mathrm{P} 6, \mathrm{P} 8$ and $\mathrm{P9}$, inequality constraint (greater than or equal and less than or equal in the same problem) through P4 and P10. Finally, the numerical examples present one type of inequality constraints greater than or equal in P5 and less than or equal constraints in P7. The result presents the difference between algebraic method [22] and the presented Algorithms. The following numerical examples denote the capacity $\mathrm{x}_{-} \mathrm{j}$ as decision variables that affect objective function (cost function) such as delivery cost in benchmark P2 and $\mathrm{P} 3$ and taxes in benchmark P1, P4... etc. The new proposal algorithms obtain the value of $\mathrm{x} \_j$ to optimize the objective function in the pessimistic case (maximum of minimum cost function). If the cost function value is less than zero, the cost is considered equal zero.
P1:

$F(X)=\max _{\mathrm{j} \in \mathrm{J}}\left(\min f_{j}\left(x_{j}\right)\right)$ Where $j=\{1,2, \ldots, 7\}$

$f_{1}\left(x_{1}\right)=-0.2057 x_{1}+1.451$,

$f_{2}\left(x_{2}\right)=4.8742 x_{2}+1.5346$,

$f_{3}\left(x_{3}\right)=2.8848 x_{3}+(-3.6121)$,

$f_{4}\left(x_{4}\right)=0.9861 x_{4}+(-0.9143)$,

$f_{5}\left(x_{5}\right)=1.7238 x_{5}+(-2.0145)$.

$f_{6}\left(x_{6}\right)=1.1737 x_{6}+1.9373$

$f_{7}\left(x_{7}\right)=-3.3199 x_{7}+(-4.8467)$

\section{Subject to:}

$\max \left(6.1221 \wedge x_{1}, 9.0983 \wedge x_{2}, 9.5032 \wedge x_{3}, 6.0123 \wedge x_{4}\right.$, $\left.6.1112 \wedge x_{5}, 4.1221 \wedge x_{6}, 5.5776 \wedge x_{7}\right) \quad=6.1221$

$\max \left(8.2984 \wedge x_{1}, 3.392 \wedge x_{2}, 2.5185 \wedge x_{3}, 1.1925 \wedge x_{4}\right.$, $\left.8.9742 \wedge x_{5}, 6.7594 \wedge x_{6}, 8.6777 \wedge x_{7}\right) \quad=7.0955$ $\max \left(2.0115 \wedge x_{1}, 6.3539 \wedge x_{2}, 4.4317 \wedge x_{3}\right.$, $\left.7.7452 \wedge x_{4}, 0.6465 \wedge x_{5}, 9.4098 \wedge x_{6}, 1.3576 \wedge x_{7}\right)=$ 6.3539

$\max \left(6.4355 \wedge x_{1}, 1.6404 \wedge x_{2}, 3.185 \wedge x_{3}, 3.7361 \wedge x_{4}\right.$, $\left.7.2605 \wedge x_{5}, 3.0201 \wedge x_{6}, 5.3808 \wedge x_{7}\right)=6.4355$

$\max \left(8.5668 \wedge x_{1}, 5.831 \wedge x_{2}, 2.5146 \wedge x_{3}, 8.7804 \wedge x_{4}\right.$, $\left.3.7709 \wedge x_{5}, 4.477 \wedge x_{6}, 2.3007 \wedge x_{7}\right) \quad=6.5712$ $\max \left(5.2690 \wedge x_{1}, 9.69 \wedge x_{2}, 5.1598 \wedge x_{3}, 9.2889 \wedge x_{4}\right.$, $\left.6.1585 \wedge x_{5}, 1.0786 \wedge x_{6}, 7.0121 \wedge x_{7}\right) \quad=7.0121$

$X l \leq X \leq X u$

$l X=(0,0,0,0,0,0,0), u X=(10,10,10,10,10,10$, 10)

P2:

$F(X)=\max _{\mathrm{j} \in \mathrm{J}}\left(\min f_{j}\left(x_{j}\right)\right)$ where $j=\{1,2, \ldots, 6\}$

$f_{1}\left(x_{1}\right)=\left(x_{1}-3.3529\right)^{2}$

$f_{2}\left(x_{2}\right)=\left(x_{2}-1.4656\right)^{2}$

$f_{3}\left(x_{3}\right)=\left(x_{3}-5.6084\right)^{2}$

$f_{4}\left(x_{4}\right)=\left(x_{4}-5.6532\right)^{2}$

$f_{5}\left(x_{5}\right)=\left(x_{5}-6.1536\right)^{2}$

$f_{6}\left(x_{6}\right)=\left(x_{6}-6.5893\right)^{2}$

\section{Subject to:}

$\max \left(3.694 \wedge x_{1}, 0.874 \wedge x_{2}, 0.5518 \wedge x_{3}, 4.6963 \wedge\right.$ $\left.x_{4}, 2.123 \wedge x_{5}, 1.4673 \wedge x_{6}\right) \quad=4.0195$

$\max \left(1.9585 \wedge x_{1}, 8.347 \wedge x_{2}, 5.815 \wedge x_{3}, 8.5545 \wedge x_{4}\right.$, $\left.8.9532 \wedge x_{5}, 8.7031 \wedge x_{6}\right)=7.2296$

$\max \left(1.3207 \wedge x_{1}, 8.961 \wedge x_{2}, 1.5718 \wedge x_{3}, 3.7155 \wedge x_{4}\right.$, $\left.0.1555 \wedge x_{5}, 4.3611 \wedge x_{6}\right)=4.2766$

$\max \left(8.4664 \wedge x_{1}, 9.1324 \wedge x_{2}, 6.6594 \wedge x_{3}, 2.5637 \wedge x_{4}\right.$, $\left.6.0204 \wedge x_{5}, 6.0846 \wedge x_{6}\right)=6.6594$

$\max \left(2.4219 \wedge x_{1}, 9.6081 \wedge x_{2}, 1.9312 \wedge x_{3}, 2.5218 \wedge x_{4}\right.$, $\left.1.3976 \wedge x_{5}, 4.1969 \wedge x_{6}\right) \quad=4.1969$

$\max \left(1.1172 \wedge x_{1}, 3.6992 \wedge x_{2}, 7.5108 \wedge x_{3}, 4.7686 \wedge x_{4}\right.$, $\left.4.4845 \wedge x_{5}, 4.3301 \wedge x_{6}\right)=6.9784$

$X l \leq X \leq X u$

$l X=(0,0,0,0,0,0), u X=(10,10,10,10,10,10)$ 
P3:

$F(X)=\max _{j \in J}\left(\min f_{j}\left(x_{j}\right)\right)$ where $j=\{1,2, \ldots, 6\}$

$f_{1}\left(x_{1}\right)=\left|\left(x_{1}-3.3529\right)\left(x_{1}-0.7399\right)\right|$,

$f_{2}\left(x_{2}\right)=\left|\left(x_{2}-1.4656\right)\left(x_{2}+0.1385\right)\right|$,

$f_{3}\left(x_{3}\right)=\left|\left(x_{3}-5.6084\right)\left(x_{3}+4.1585\right)\right|$,

$f_{4}\left(x_{4}\right)=\left|\left(x_{4}-5.6532\right)\left(x_{4}-1.1625\right)\right|$,

$f_{5}\left(x_{5}\right)=\left|\left(x_{5}-6.1536\right)\left(x_{5}+2.0188\right)\right|$,

$f_{6}\left(x_{6}\right)=\left|\left(x_{6}-6.5893\right)\left(x_{6}-1.2852\right)\right|$.

\section{Subject to:}

$\max \left(3.694 \wedge x_{1}, 0.874 \wedge x_{2}, 0.5518 \wedge x_{3}, 4.6963 \wedge x_{4}\right.$, $\left.2.123 \wedge x_{5}, 1.4673 \wedge x_{6}\right)=4.0195$

$\max \left(1.958 \wedge x_{1}, 8.347 \wedge x_{2}, 5.815 \wedge x_{3}, 8.5545 \wedge x_{4}\right.$, $\left.8.9532 \wedge x_{5}, 8.7031 \wedge x_{6}\right)=7.2296$

$\max \left(1.3207 \wedge x_{1}, 8.961 \wedge x_{2}, 1.5718 \wedge x_{3}, 3.7155 \wedge x_{4}\right.$,

$\left.0.1555 \wedge x_{5}, 4.3611 \wedge x_{6}\right)=4.2766$

$\max \left(8.4664 \wedge x_{1}, 9.1324 \wedge x_{2}, 6.6594 \wedge x_{3}, 2.5637 \wedge x_{4}\right.$,

$\left.6.6594 \wedge x_{5}, 6.0846 \wedge x_{6}\right)=6.6594$

$\max \left(2.4219 \wedge x_{1}, 9.6081 \wedge x_{2}, 1.9312 \wedge x_{3}, 2.5218 \wedge x_{4}\right.$,

$\left.1.3976 \wedge x_{5}, 4.1969 \wedge x_{6}\right)=4.1969$

$\max \left(1.1172 \wedge x_{1}, 3.6992 \wedge x_{2}, 7.5108 \wedge x_{3}, 4.7686 \wedge x_{4}\right.$, $\left.6.9874 \wedge x_{5}, 4.3301 \wedge x_{6}\right)=6.9784$

$X l \leq X \leq X u$

$l X=(0,0,0,0,0,0), u X=(10,10,10,10,10,10)$

P4:

$F(X)=\max _{\mathrm{j} \in \mathrm{J}}\left(\min f_{j}\left(x_{j}\right)\right)$

Where $j=\{1,2,3\}$

$f_{1}\left(x_{1}\right)=0.5 x_{1}+1.4$

$f_{2}\left(x_{2}\right)=0.8 x_{2}+5.2$,

$f_{3}\left(x_{3}\right)=0.7 x_{3}+3.1$.

\section{Subject to:}

$\max \left(7 \wedge x_{1}, 5 \wedge x_{2}, 6 \wedge x_{3}\right) \geq 6$

$\max \left(6 \wedge x_{1}, 7 \wedge x_{2}, 8 \wedge x_{3}\right) \geq 8$

$\max \left(8 \wedge x_{1}, 5 \wedge x_{2}, 4 \wedge x_{3}\right) \geq 4$

$l X \leq X \leq u X$,

$l X=(0,0,0), u X=(10,10,10)$

P5:

$F(X)=\max _{\mathrm{j} \in \mathrm{J}}\left(\min f_{j}\left(x_{j}\right)\right)$ Where $j=\{1,2,3\}$

$f_{1}\left(x_{1}\right)=\left|x_{1}-7.2\right|$

$f_{2}\left(x_{2}\right)=\left|0.3 x_{2}-1.9\right|$

$f_{3}\left(x_{3}\right)=\left|1.5 x_{3}-3.2\right|$

\section{Subject to:}

$\max \left(5 \wedge x_{1}, 16 \wedge x_{2}, 9 \wedge x_{3}\right) \leq 6$ $\max \left(3 \wedge x_{1}, 5 \wedge x_{2}, 8 \wedge x_{3}\right) \leq 5$ $\max \left(15 \wedge x_{1}, 7 \wedge x_{2}, 11 \wedge x_{3}\right)$ $\max \left(9 \wedge x_{1}, 8 \wedge x_{2}, 13 \wedge x_{3}\right)$ $\max \left(11 \wedge x_{1}, 3 \wedge x_{2}, 3 \wedge x_{3}\right)$ $\max \left(5 \wedge x_{1}, 6 \wedge x_{2}, 9 \wedge x_{3}\right) \geq$ $\max \left(5 \wedge x_{1}, 3 \wedge x_{2}, 12 \wedge x_{3}\right) \quad \geq 4$ $\max \left(3 \wedge x_{1}, 5 \wedge x_{2}, 9 \wedge x_{3}\right) \geq 2$ $\max \left(5 \wedge x_{1}, 5 \wedge x_{2}, 6 \wedge x_{3}\right) \geq 5$ $l X \leq X \leq u X, l X=(0,0,0), u X=(10,10,10)$

P6:

$F(X)=\max _{\mathrm{j} \in \mathrm{J}}\left(\min f_{j}\left(x_{j}\right)\right)$ Where $j=\{1,2,3\}$

$f_{1}\left(x_{1}\right)=\left(x_{1}-4\right)^{2}+1.5$

$f_{2}\left(x_{2}\right)=\left(x_{2}-9\right)^{2}+2.5$

$f_{3}\left(x_{3}\right)=\left(x_{3}-16\right)^{2}+5.5$

\section{Subject to}

$\max \left(9 \wedge x_{1}, 14 \wedge x_{2}, 5 \wedge x_{3}\right)=9$

$\max \left(3 \wedge x_{1}, 4 \wedge x_{2}, 7 \wedge x_{3}\right)=7$

$\max \left(15 \wedge x_{1}, 8 \wedge x_{2}, 7 \wedge x_{3}\right)=8$

$\max \left(11 \wedge x_{1}, 25 \wedge x_{2}, 10 \wedge x_{3}\right) \leq 20$

$\max \left(15 \wedge x_{1}, 12 \wedge x_{2}, 5 \wedge x_{3}\right) \leq 15$

$\max \left(7 \wedge x_{1}, 6 \wedge x_{2}, 5 \wedge x_{3}\right) \leq 10$

$\max \left(32 \wedge x_{1}, 5 \wedge x_{2}, 4 \wedge x_{3}\right) \quad \geq 5$

$\max \left(12 \wedge x_{1}, 10 \wedge x_{2}, 5 \wedge x_{3}\right) \quad \geq 7$

$\max \left(20 \wedge x_{1}, 17 \wedge x_{2}, 30 \wedge x_{3}\right) \quad \geq 6$

$l X \leq X \leq u X, l X=(0,0,0), u X=(30,30,30)$

P7:

$F(X)=\max _{\mathrm{j} \in \mathrm{J}}\left(\min f_{j}\left(x_{j}\right)\right)$

Where $j=\{1,2,3,4,5\}$

$f_{1}\left(x_{1}\right)=\left|\left(x_{1}-5\right)\left(x_{1}-7\right)\right|$,

$f_{2}\left(x_{2}\right)=\left|\left(x_{2}-10\right)\left(x_{2}-12\right)\right|$,

$f_{3}\left(x_{3}\right)=\left|\left(x_{3}-1\right)\left(x_{3}-2\right)\right|$,

$f_{4}\left(x_{4}\right)=\left|\left(x_{4}-2.5\right)\left(x_{4}-5.5\right)\right|$,

$f_{5}\left(x_{5}\right)=\left|\left(x_{5}-1\right)\left(x_{5}-14\right)\right|$.

\section{Subject to}

$\max \left(55.1 \wedge x_{1}, 10.5 \wedge x_{2}, 20.3 \wedge x_{3}, 51.1 \wedge x_{4}, 18.2 \wedge\right.$

$\left.x_{5}\right) \quad \leq 12.7$

$\max \left(26.2 \wedge x_{1}, 22.2 \wedge x_{2}, 19.3 \wedge x_{3}, 65.5 \wedge x_{4}, 55.2 \wedge\right.$

$\left.x_{5}\right) \quad \leq 18.8$

$\max \left(2.5 \wedge x_{1}, 17.3 \wedge x_{2}, 11 \wedge x_{3}, 5.1 \wedge x_{4}, 18.3 \wedge x_{5}\right)$ $\leq 11.2$

$l X \leq X \leq u X$

$l X=(0,0,0,0,0), u X=(20,20,20,20,20)$

P8:

$F(X)=\max _{\mathrm{j} \in \mathrm{J}}\left(\min f_{j}\left(x_{j}\right)\right)$

Where $j=\{1,2, \ldots, 6\}$

$f_{1}\left(x_{1}\right)=\left|\left(x_{1}-4\right)\right|$,

$f_{2}\left(x_{2}\right)=\left|\left(x_{2}-2.5\right)\right|$,

$f_{3}\left(x_{3}\right)=\left|\left(x_{3}-3\right)\right|$,

$f_{4}\left(x_{4}\right)=\left|\left(x_{4}-1\right)\right|$

$f_{5}\left(x_{5}\right)=\left|\left(x_{5}-1.5\right)\right|$, 
$f_{6}\left(x_{6}\right)=\left|\left(x_{6}-6\right)\right|$.

\section{Subject to:}

$\max \left(15 \wedge x_{1}, 20 \wedge x_{2}, 22 \wedge x_{3}, 7 \wedge x_{4}, 15 \wedge x_{5}, 30 \wedge x_{6}\right)=7$ $\max \left(6 \wedge x_{1}, 25 \wedge x_{2}, 22 \wedge x_{3}, 30 \wedge x_{4}, 35 \wedge x_{5}, 31 \wedge x_{6}\right)=6$ $\max \left(10 \wedge x_{1}, 20 \wedge x_{2}, 15 \wedge x_{3}, 18 \wedge x_{4}, 12 \wedge x_{5}, 9 \wedge x_{6}\right) \geq 5$ $\max \left(10 \wedge x_{1}, 8 \wedge x_{2}, 20 \wedge x_{3}, 12 \wedge x_{4}, 11 \wedge x_{5}, 17 \wedge x_{6}\right) \leq 9$ $l X \leq X \leq u X$,

$l X=(0,0,0,0,0,0), u X=(10,10,10,10,10,10)$

\section{P9:}

$$
\begin{aligned}
& F(X)=\max _{\mathrm{j} \in \mathrm{J}}\left(\min f_{j}\left(x_{j}\right)\right) \\
& \quad \text { Where } j=\{1,2, \ldots, 5\} \\
& f_{1}\left(x_{1}\right)=\left|\left(x_{1}-2\right)\left(x_{1}-5\right)\right| \\
& f_{2}\left(x_{2}\right)=\left|\left(x_{2}^{2}-4\right)\right|+0.5 \\
& f_{3}\left(x_{3}\right)=\left|\left(x_{3}-2.5\right)\left(x_{3}-4.25\right)\right|, \\
& f_{4}\left(x_{4}\right)=\left|\left(x_{4}^{2}-6.5\right)\right|+0.5, \\
& f_{5}\left(x_{5}\right)=\left|\left(x_{5}-9\right)\left(x_{5}-10\right)\right| .
\end{aligned}
$$

\section{Subject to}

$\max \left(14 \wedge x_{1}, 20 \wedge x_{2}, 6 \wedge x_{3}, 12 \wedge x_{4}, 2 \wedge x_{5}\right) \quad=12.7$ $\max \left(30 \wedge x_{1}, 13 \wedge x_{2}, 12 \wedge x_{3}, 10 \wedge x_{4}, 8 \wedge x_{5}\right)=8$ $\max \left(3 \wedge x_{1}, 10 \wedge x_{2}, 13 \wedge x_{3}, 21 \wedge x_{4}, 15 \wedge x_{5}\right)=10$ $\max \left(15 \wedge x_{1}, 21 \wedge x_{2}, 18 \wedge x_{3}, 25 \wedge x_{4}, 30 \wedge x_{5}\right) \leq 20$ $\max \left(30 \wedge x_{1}, 21 \wedge x_{2}, 38 \wedge x_{3}, 40 \wedge x_{4}, 15 \wedge x_{5}\right) \leq 35$ $\max \left(15 \wedge x_{1}, 19 \wedge x_{2}, 11 \wedge x_{3}, 18 \wedge x_{4}, 20 \wedge x_{5}\right) \geq 10$ $\max \left(22 \wedge x_{1}, 30 \wedge x_{2}, 15 \wedge x_{3}, 25 \wedge x_{4}, 30 \wedge x_{5}\right) \geq 8$ $l X \leq X \leq u X$ $l X=(0,0,0,0,0), u X=(20,20,20,20,20)$

\section{P10:}

$$
\begin{aligned}
& F(X)=\max _{\mathrm{j} \in \mathrm{J}}\left(\min f_{j}\left(x_{j}\right)\right) \\
& \text { Where } j=\{1,2, \ldots, 5\} \\
& f_{1}\left(x_{1}\right)=\left|\sin \left(x_{1}\right)+0.2\right|+0.2 \\
& f_{2}\left(x_{2}\right)=\left|\sin \left(x_{2}+1\right)+0.3\right|+0.3 \\
& f_{3}\left(x_{3}\right)=\left|\sin \left(x_{3}+2\right)+0.4\right|+0.4 \\
& f_{4}\left(x_{4}\right)=\left|\sin \left(x_{4}+3\right)+0.5\right|+0.5 \\
& f_{5}\left(x_{5}\right)=\left|\sin \left(x_{5}+4\right)+0.6\right|+0.6 ;
\end{aligned}
$$

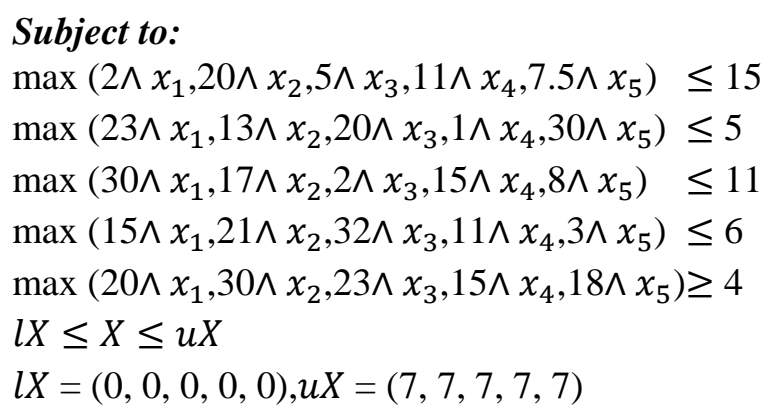

The propose algorithms are applied by software Matlab R2013a and executed by hardware Intel (R) core $^{\mathrm{TM}} \mathrm{i} 5$ 2520M CPU. All algorithms were repeated $(t)$ number of iteration $=100$. After examining several numbers of iteration we get the suitable number of iterations $=100$ to reach the optimal solutions. Moreover, we tested the number of particle swarm $n p o p=20,30$ and 40 to find the suitable number of particle swarm npop $=30$ with parameter $(\mathrm{c} 1, \mathrm{c} 2)=$ $(2,2)$, the inertia weight $w \in[0.1,1]$. The algorithms MNS-PSO-GA and MNS-PSO-GA-cross were applied by crossover rate $75 \%$ according to recommended [28] and the MNS-PSO-GA and MNS-PSO-GA-Mut were applied by mutation rate $5 \%[28,29]$ which is suitable according to the size of particle swarmnpop. The benchmark functions were tested through P1 to P5 and investigated numerical example P6 which has all types of constraints such as Eq.(2), Eq.(3) and Eq.(4). The results are presented after 100 feasible trials and calculated the best result which is considered as the minimum result of feasible trials, average results and standard deviation (S.D).

The starting of all proposal algorithms is applied the MPA algorithm to determine the feasible set of solution then search the optimal solutions with in the feasible region, see Table. 2.

The MNS-PSO algorithm and MNS-LAPSO algorithm could reach the optimal solution as an algebraic method in regards to the best solution for all tested numerical examples except P2 which isbetter than the Algebraic Method, see Table 3. and Fig. 1.

On the other hand, the (MNS-PSO-GA-Mut) algorithm can reach optimal solution the same as algebraic Method in regard to the best result for all numerical examples except $\mathrm{P} 2$ which is better than Algebraic Method. Also, the better result can be obtained for P6 in regard to the best result, see Table 3. and Fig. 1.

The three types of hybrid PSO are: - the first is (MNS-PSO-GA) algorithm which has two processes crossover and mutation. The second is (MNS-PSOGA-Cross) algorithm which has crossover process only and the third is (MNS-PSO-GA-Mut) algorithm which has mutation process only. These algorithms improve algorithms improve the solution. The (MNSPSO-GA) and (MNS-PSO-GA-Cross) Algorithms can reach the optimal solution the same as algebraic method in regard of the best result for all numerical examples except P1, P2 and P3 which are better than the Algebraic Method. Moreover, the (MNS-PSOGA) only improved the solution in regard of the best result of P8 and P9 see see Table 3. and Fig. 1. 
Table 2. The new upper limit of numerical examples which are found by Max-Point Algorithm

\begin{tabular}{|l|l|}
\hline P1 & $\begin{array}{l}u x(\text { new })= \\
{[6.5712,6.1221,6.1221,6.3539,6.4355,6.3539,7.09} \\
55]\end{array}$ \\
\hline P2 & $\begin{array}{l}u x(\text { new })= \\
{[6.6594,4.1969,6.9874,4.0195,7.2296,4.2766]}\end{array}$ \\
\hline P3 & $\begin{array}{l}u x(\text { new })= \\
{[6.6594,4.1969,6.9874,4.0195,7.2296,4.2766]}\end{array}$ \\
\hline P4 & $u x($ new $)=[10,10,10]$ \\
\hline P5 & $u x($ new $)=[7,6,5]$ \\
\hline P6 & $u x($ new $)=[8,9,30]$ \\
\hline P7 & $u x($ new $)=[12.7,11.2,12.7,12.7,11.2]$ \\
\hline P8 & $u x($ new $)=[7,6,6,6,6,6]$ \\
\hline P9 & $u x($ new $)=[6,6,8,6,10]$ \\
\hline P10 & $u x($ new $)=[5,5,5,6,5]$ \\
\hline
\end{tabular}

All proposal Algorithms could obtain multioptimal solutions and multi-values of decision variables in P3 are $x_{(1)}^{*}$ and $x_{(2)}^{*}$ for multi-model objective

functions such as P3 are

(3.3529,1.4656,5.6084,4.0195,7.2296,4.2766),

$(0.7399,1.4656,5.6084,4.0195,7.2296,4.2766)$

respectively. The algebraic method obtained one solution only $x^{*}=(3.3297,1.4689,6.9874,4.0195$, $7.2296,4.2766)$. Also, in P7 the proposal algorithms obtain 8 optimal solutions $x_{(1)}^{*}$ to $x_{(8)}^{*}$ and in P10 the proposal algorithms obtain 4 optimal solutions $x_{(1)}^{*}$ to $x_{(4)}^{*}$ which are presented the same result of objective function but the algebraic method presents only one solution [22], see Table 2 .

The MNS-PSO-GA algorithm got a better optimal result of separation objective functions $\min f_{j}\left(x_{j}\right)$ where $j=\{1,2,3\}$ are $(1.4,5.2,8.7)$ and $(0.2,0.1,0)$ for P4 and P5 respectively with corresponding decision variables are $x^{*}=(0,0,8)$ and $x^{*}=(7,6,2.1333)$ respectively with the same value of $\mathrm{F}(\mathrm{X})$ of $\mathrm{P} 4$ and $\mathrm{P} 5$ are 8.7 and 0.2 , respectively. On the other hand the algebraic method get the same values of objective function of $F(X)$ for P4 and P5 with different values of $\min f_{j}\left(x_{j}\right)$ are $(4.4,5.2,8.7)$ and $(0.2,0.1,0.02)$ respectively with corresponding decision variables $x^{*}=(6,0,8)$ and $x^{*}=(7,6,2.12)$ respectively, see Table 2 . The results of presented algorithms optimize the separated objective functions $\min f_{j}\left(x_{j}\right)$, then maximize the result. Moreover, the presented algorithms will become suitable for solving min-min separating problem or the need of decision maker to calculate the range of objective function result in case of max-min separate problem (pessimistic case) and min-min separate problem (Optimistic case).
Generally, the (MNS-PSO-GA) Algorithm achieves the best average of all testing algorithms for all benchmark functions except P7, P9 and P10 the best average of P7 and P10 was found by (MNSLOAPSO) and the best average of $\mathrm{P} 9$ was found by MNS-PSO algorithm, see Fig. 2.

\section{Conclusion}

The (MMSMMLC) problem included the max-min Algebraic in objective function and constraints to solve economics problem and control the recourses in the huge and complex network such as transportation network, water network, electricity network, etc. This paper presents other methods for solving

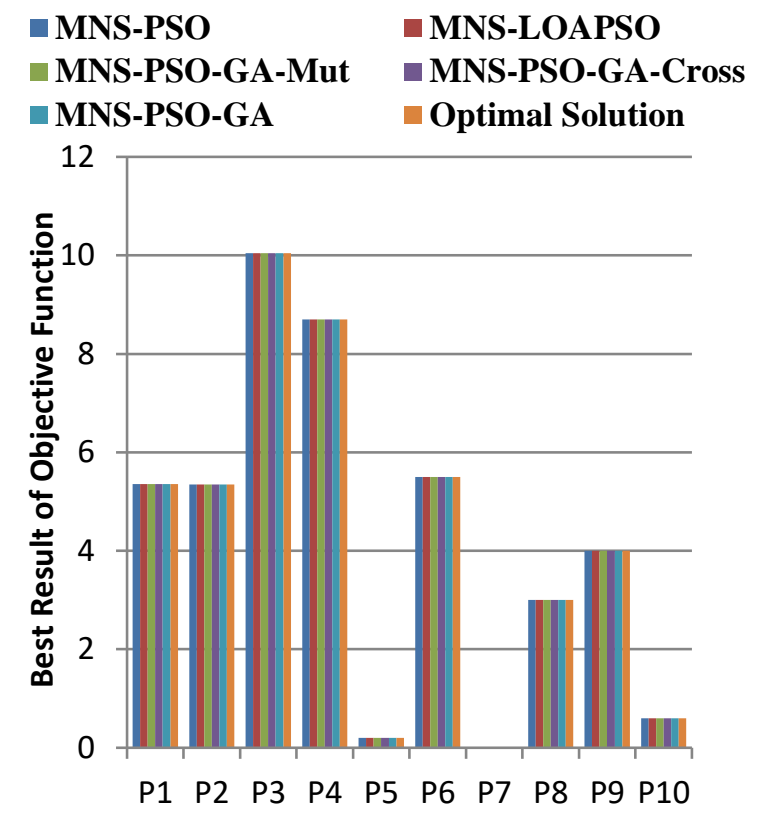

Figure. 1 The Best Result of $F(X)$ for each numerical example and optimal solution of Algebraic method

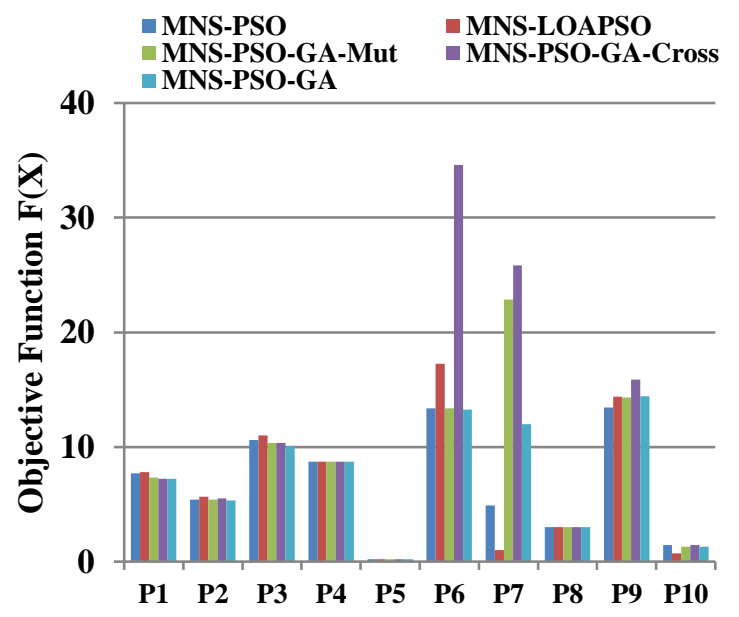

Figure. 2 The average $F(X)$ of each numerical example 
Table 3. Comparison between Algebraic Method, MNS-PSO, MNS-LOAPSO, MNS-PSO-GA-Mut, MNS-PSO-GACross and MNS-PSO-GA after 100 independent feasible runs where SD is the standard deviation

\begin{tabular}{|c|c|c|c|c|c|c|c|}
\hline $\begin{array}{l}\text { Bench } \\
\text { mark }\end{array}$ & $\begin{array}{c}\text { Type of } \\
\text { Constraints }\end{array}$ & $\begin{array}{l}\text { Objective } \\
\text { Function }\end{array}$ & Algorithms & $\begin{array}{c}\text { Best } \\
\text { result }\end{array}$ & Average & S.D & Optimal \\
\hline \multirow{6}{*}{$\mathrm{P} 1$} & \multirow{6}{*}{ Equality } & \multirow{6}{*}{$\begin{array}{l}\text { Uni- } \\
\text { modal }\end{array}$} & Algebraic Method & & & & 5.3513 \\
\hline & & & MNS-PSO & 5.35128 & 7.69721 & 3.14 & \\
\hline & & & MNS-LOAPSO & 5.35128 & 7.78874 & 3.12 & \\
\hline & & & MNS-PSO-GA-Mut & 5.35128 & 7.33264 & 2.03 & \\
\hline & & & MNS-PSO-GA-Cross & 5.35118 & 7.21128 & 2.03 & \\
\hline & & & MNS-PSO-GA & 5.35118 & 7.21128 & 2.02 & \\
\hline \multirow{6}{*}{$\mathrm{P} 2$} & \multirow{6}{*}{ Equality } & \multirow{6}{*}{$\begin{array}{l}\text { Uni- } \\
\text { modal }\end{array}$} & Algebraic Method & & & & 5.3488 \\
\hline & & & MNS-PSO & 5.34858 & 5.404424 & 0.56 & \\
\hline & & & MNS-LOAPSO & 5.34858 & 5.656414 & 1.16 & \\
\hline & & & MNS-PSO-GA-Mut & 5.34858 & 5.40442 & 0.56 & \\
\hline & & & MNS-PSO-GA-Cross & 5.34858 & 5.51611 & 0.96 & \\
\hline & & & MNS-PSO-GA & 5.34858 & 5.34858 & $1.4 \times 10^{-14}$ & \\
\hline \multirow{6}{*}{ P3 } & \multirow{6}{*}{ Equality } & \multirow{6}{*}{$\begin{array}{l}\text { Multi- } \\
\text { modal }\end{array}$} & Algebraic Method & & & & 10.0481 \\
\hline & & & MNS-PSO & 10.04812 & 10.61116 & 1.87 & \\
\hline & & & MNS-LOAPSO & 10.04812 & 11.00449 & 2.42 & \\
\hline & & & MNS-PSO-GA-Mut & 10.04812 & 10.35625 & 1.4 & \\
\hline & & & MNS-PSO-GA-Cross & 10.04708 & 10.33334 & 1.63 & \\
\hline & & & MNS-PSO-GA & 10.04708 & 10.04756 & $5.2 \times 10^{-4}$ & \\
\hline \multirow{6}{*}{$\mathrm{P} 4$} & \multirow{6}{*}{$\begin{array}{l}\text { Inequality } \\
\qquad(\geq \leq)\end{array}$} & \multirow{6}{*}{$\begin{array}{l}\text { Uni- } \\
\text { modal }\end{array}$} & Algebraic Method & & & & 8.7 \\
\hline & & & MNS-PSO & 8.7 & 8.7 & $2.8 \times 10^{-9}$ & \\
\hline & & & MNS-LOAPSO & 8.7 & 8.7 & $9.5 \times 10^{-12}$ & \\
\hline & & & MNS-PSO-GA-Mut & 8.7 & 8.7 & $1.6 \times 10^{-14}$ & \\
\hline & & & MNS-PSO-GA-Cross & 8.7 & 8.7 & $1.9 \times 10^{-12}$ & \\
\hline & & & MNS-PSO-GA & 8.7 & 8.7 & $2.1 \times 10^{-12}$ & \\
\hline \multirow{6}{*}{ P5 } & \multirow{6}{*}{$\begin{array}{l}\text { Inequality } \\
(\geq) \text { only }\end{array}$} & \multirow{6}{*}{$\begin{array}{l}\text { Uni- } \\
\text { modal }\end{array}$} & Algebraic Method & & & & 0.2 \\
\hline & & & MNS-PSO & 0.2 & 0.2 & $5.3 \times 10^{-16}$ & \\
\hline & & & MNS-LOAPSO & 0.2 & 0.2 & $5.3 \times 10^{-16}$ & \\
\hline & & & MNS-PSO-GA-Mut & 0.2 & 0.2 & $5.3 \times 10^{-16}$ & \\
\hline & & & MNS-PSO-GA-Cross & 0.2 & 0.2 & $5.3 \times 10^{-16}$ & \\
\hline & & & MNS-PSO-GA & 0.2 & 0.2 & $5.3 \times 10^{-16}$ & \\
\hline \multirow{6}{*}{ P6 } & \multirow{6}{*}{$\begin{array}{c}\text { Inequality }(\geq, \leq) \\
\text { and equality }\end{array}$} & \multirow{6}{*}{$\begin{array}{l}\text { Uni- } \\
\text { modal }\end{array}$} & Algebraic Method & & & & 5.5 \\
\hline & & & MNS-PSO & 5.5 & 13.38 & 38.2 & \\
\hline & & & MNS-LOAPSO & 5.5 & 17.24 & 42.26 & \\
\hline & & & MNS-PSO-GA-Mut & 5.5 & 13.38 & 38.2 & \\
\hline & & & MNS-PSO-GA-Cross & 5.5 & 34.6 & 69.62 & \\
\hline & & & MNS-PSO-GA & 5.5 & 13.26 & 38.2 & \\
\hline \multirow{6}{*}{ P7 } & \multirow{6}{*}{$\begin{array}{l}\text { Inequality } \\
(\leq) \text { only }\end{array}$} & \multirow{6}{*}{$\begin{array}{l}\text { Multi- } \\
\text { modal }\end{array}$} & Algebraic Method & & & & 0 \\
\hline & & & MNS-PSO & $\begin{array}{c}2.887 \times \\
10^{-15} \\
\end{array}$ & 4.884 & 10.77 & \\
\hline & & & MNS-LOAPSO & $\begin{array}{c}9.814 \times \\
10^{-14} \\
\end{array}$ & 1.001 & 4.883 & \\
\hline & & & MNS-PSO-GA-Mut & $\begin{array}{c}2.887 \times \\
10^{-15} \\
\cong 0\end{array}$ & 22.86 & 11.46 & \\
\hline & & & MNS-PSO-GA-Cross & $\begin{array}{c}8.156 \times \\
10^{-11} \\
\cong 0\end{array}$ & 25.84 & 34.96 & \\
\hline & & & MNS-PSO-GA & $\begin{array}{c}2.664 \times \\
10^{-11} \\
\cong 0\end{array}$ & 12 & 14.17 & \\
\hline \multirow{3}{*}{ P8 } & & & Algebraic Method & & & & 3 \\
\hline & Inequality $(\geq, \leq)$ & Un1- & MNS-PSO & 3 & 3 & 0 & \\
\hline & & & MNS-LOAPSO & 3 & 3 & 0 & \\
\hline
\end{tabular}




\begin{tabular}{|c|c|c|c|c|c|c|c|}
\hline & & & MNS-PSO-GA-Mut & 3 & 3 & 0 & \\
\hline & & & MNS-PSO-GA-Cross & 3 & 3.015 & 0.15 & \\
\hline & & & MNS-PSO-GA & 2.99999 & 3 & $\begin{array}{c}3.015 \times \\
10^{-5}\end{array}$ & \\
\hline \multirow{6}{*}{ P9 } & \multirow{6}{*}{$\begin{array}{c}\text { Inequality }(\geq, \leq) \\
\text { and equality }\end{array}$} & \multirow{6}{*}{$\begin{array}{l}\text { Multi- } \\
\text { modal }\end{array}$} & Algebraic Method & & & & 4 \\
\hline & & & MNS-PSO & 4 & 13.45 & 12.01 & \\
\hline & & & MNS-LOAPSO & 4 & 14.38 & 12.45 & \\
\hline & & & MNS-PSO-GA-Mut & 4 & 14.3 & 12.36 & \\
\hline & & & MNS-PSO-GA-Cross & 4 & 15.86 & 12.99 & \\
\hline & & & MNS-PSO-GA & 3.9995 & 14.44 & 12.47 & \\
\hline \multirow{6}{*}{$\mathrm{P} 10$} & \multirow{6}{*}{$\begin{array}{l}\text { Inequality } \\
\qquad(\geq \leq)\end{array}$} & \multirow{6}{*}{$\begin{array}{l}\text { Multi- } \\
\text { modal }\end{array}$} & Algebraic Method & & & & 0.6 \\
\hline & & & MNS-PSO & 0.6 & 1.456 & 0.3093 & \\
\hline & & & MNS-LOAPSO & 0.6 & 0.7179 & 0.2923 & \\
\hline & & & MNS-PSO-GA-Mut & 0.6 & 1.295 & 0.4263 & \\
\hline & & & MNS-PSO-GA-Cross & 0.6 & 1.449 & 0.3251 & \\
\hline & & & MNS-PSO-GA & 0.6 & 1.309 & 0.426 & \\
\hline
\end{tabular}

Table 2. the results of objective function $\mathrm{F}(\mathrm{X})$ and decision variable $x^{*}$ using Algebraic Method and MNS-

\begin{tabular}{|c|c|c|}
\hline \multicolumn{3}{|c|}{ PSO-GA } \\
\hline & Algebraic Method & MNS-PSO-GA \\
\hline P1 & $\begin{array}{l}F(X)=\max (0.1039, \\
1.5346,-3.6121, \\
\mathbf{5 . 3 5 1 3},-2.0145, \\
1.9373,-28.4031) \\
x^{*}=6.5712,0,0,6.353 \\
9,0,0,7.0955)\end{array}$ & $\begin{array}{l}F(X)=\max (0.10398, \\
1.5346,- \\
3.6121, \mathbf{5 . 3 5 1 1 8},- \\
2.0145,1.9373,- \\
28.40305) \\
x^{*}=(6.5712,0, \\
0,6.3538,0,0, \\
7.0955)\end{array}$ \\
\hline$P 2$ & $\begin{array}{l}F(X)= \\
\max (0,0,1.90164,2.66 \\
898,1.15778,5.3488) \\
x^{*}= \\
(3.3297,1.4689,6.9874 \\
, 4.0195,7.2296,4.2766\end{array}$ & $\begin{array}{l}F(X)= \\
\max (0, \cong 0,1.901641,2 . \\
66898,1.15778,5 \cdot 3485 \\
\mathbf{8 8}) \\
x^{*}= \\
(3.3529,1.4656,6.9874, \\
4.0195,7.2296,4.2766)\end{array}$ \\
\hline$P 3$ & $\begin{array}{l}F(X)= \\
\max (0,0,0,4.667, \mathbf{1 0 . 0 4} \\
\mathbf{8 1}, 6.9182) \\
x^{*}=(0.7325,1.4689,5 . \\
5899,4.0195,7.2296,4 . \\
2766)\end{array}$ & $\begin{array}{l}F(X)= \\
\max (\cong 0, \cong 0, \cong 0,4.667 \\
488, \cong \mathbf{1 0 . 0 4 8 1 2}, 6.918 \\
218) \\
x_{(1)}^{*}= \\
(3.3529,1.4656,5.6084, \\
4.0195,7.2296,4.2766) \\
x_{(2)}^{*}= \\
(0.7399,1.4656,5.6084, \\
4.0195,7.2296,4.2766)\end{array}$ \\
\hline $\mathrm{P} 4$ & $\begin{array}{l}F(X)= \\
\max (4.4,5.2,8.7) \\
x^{*}=(6,0,8)\end{array}$ & $\begin{array}{l}F(X)= \\
\max (1.4,5.2,8.7) \\
x^{*}=(0,0,8)\end{array}$ \\
\hline P5 & $\begin{array}{l}F(X)=\max (\mathbf{0 . 2}, 0.1 \\
0.02) \\
x^{*}=(7,6,2.12)\end{array}$ & $\begin{array}{l}F(X)=\max (\mathbf{0 . 2}, 0.1 \\
0) \\
x^{*}=(7,6,2.133)\end{array}$ \\
\hline P6 & $\begin{array}{l}F(X)=\max (1.5, \\
2.5, \mathbf{5 . 5}) \\
x^{*}=(4,9,16)\end{array}$ & $\begin{array}{l}F(X)=\max (1.5,2.5, \\
\mathbf{5 . 5}) \\
x^{*}=(4,9,16)\end{array}$ \\
\hline P7 & $\begin{array}{l}F(X)= \\
\max (\mathbf{0}, 0,0,0,0) \\
x^{*}=[5,10,1,2.5,1]\end{array}$ & $\begin{array}{l}F(X)=\max (\mathbf{0}, 0,0,0,0) \\
x_{(1)}^{*}=[5,10,1,2.5,1] ; \\
x_{(2)}^{*}=[7,10,1,2.5,1]\end{array}$ \\
\hline
\end{tabular}

\begin{tabular}{|l|l|l|}
\hline & & $x_{(3)}^{*}=[7,10,1,5.5,1]$ \\
& & $x_{(4)}^{*}=[5,10,1,5.5,1]$ \\
& & $x_{(5)}^{*}=[7,10,2,2.5,1]$ \\
& & $x_{(6)}^{*}=[7,10,2,5.5,1]$ \\
& & $x_{(7)}^{*}=[5,10,2,5.5,1]$ \\
& & $x_{(8)}^{*}=[5,10,2,2.5,1]$ \\
\hline P8 & $F(X)=\max (\mathbf{3}, 0,0,0$, & $F(X)=\max (\mathbf{2 . 9 9 9 9}, 0$, \\
& $0,0)$ & $0,0,0,0)$ \\
& $x^{*}=[7,2.5,3,1$, & $x^{*}=[6.9999,2.5,3$, \\
& $1.5,6]$ & $1,1.5,6]$ \\
\hline P9 & $F(X)=\max (\mathbf{4}, 0.5,0$, & $F(X)=\max (\mathbf{3 . 9 9 9 5}$, \\
& $0.5,0)$ & $0.5,0,0.5,0)$ \\
& $x^{*}=[6,2,2.5,2.5,10]$ & $x_{(1)}^{*}=[6$, \\
& & $2,2.5,2.5,10] ;$ \\
& & $x_{(2)}^{*}=[6$, \\
\hline P1 & $F(X)=\max (0.2,0.3$, & $F(X)=\max (0.2,0.3$, \\
0 & $0.4,0.5, \mathbf{0 . 6})$ & $0.4,0.5, \mathbf{0 . 6})$ \\
& $x^{*}=[3.343,4.978$, & $x_{(1)}^{*}=[3.343,4.978$, \\
& $1.553,2.76,1.64]$ & $1.553,2.76,1.64]$ \\
& & $x_{(2)}^{*}=[3.343,4.978$, \\
& & $1.553,0.665,1.64]$ \\
& & $x_{(3)}^{*}=[3.343,4.978$, \\
& & $3.8717,2.76,1.64]$ \\
& & $x_{(4)}^{*}=[3.343,4.978$, \\
& & $3.8717,0.665,1.64]$ \\
\hline & &
\end{tabular}

(MMSMMLC) problem using Hybrid-PSO algorithm under any type of constraints (inequality and/or equality). The Hybrid-PSO algorithms are (MNS-PSO-GA-Cross, MNS-PSO-GA-Mut and MNS-PSO-GA) and the modified algorithms are MNS-PSO and MNS-LAPSO compared with algebraic method [22] and are tested these algorithms through several benchmark functions. From the previous results we observed that the performance of Hybrid-PSO (MNS-PSO-GA, MNS-PSO-GA-Cross and MNS-PSO-GA-Mut) algorithms are improved the solution better than the algebraic method. All 
meta-heuristics algorithms get multi-solutions and multi values of decision variable especially in case of multi-model objective functions but the algebraic method lead to only one optimal solution with only value of decision variable. So, the proposal algorithms avoided that shortcoming.

In future work other meta-heuristic methods can be applied to solve MMSMMLC problem with stochastic variable in right hand side or left hand side to suit the real life situations. Moreover, other metaheuristics methods can be applied on the MSMMLC problem under nonlinear max-min constraints

\section{Conflicts of Interest}

The authors declare no conflict of interest.

\section{Author Contributions}

The paper conceptualization, methodology, software, validation, formal analysis, investigation, resources, data curation, writing-original draft preparation, writing-review and editing have been done by 1 st author. The supervision, methodology , validation and reviewing have been done by 2 nd author, The conceptualization, writing review have been done by 3rd author and the writing-review and editing, supervision and validation have been done by 4th author.

\section{References}

[1] R. A. Cuninghame-Green, Minimax algebra, Lecture notes in economics and mathematical systems, Vol 166. Berlin: Springer, 1979.

[2] K. Zimmermann, "Optimization problems with unimodal fnctions in max-separable constraints", Optimization, Vol. 24, No. 1-2, pp. 31-41, Jan. 1992.

[3] K.Zimmermann, "Disjunctive optimization, max-separable problems and extremal algebras", Theoretical Computer Science, Vol. 293, No. 1, pp. 45-54, 2003.

[4] A.Tharwat, and K. Zimmermann, "One class of separable optimization problems :solution method, application", Optimization, Vol. 59, No. 5, pp. 619-625, Jul. 2010.

[5] M. Gavalec, and K. Zimmermann, "Duality for max-separable problems", Central European Journal of Operations Research, Vol. 20, No. 3, pp. 409-419, Sep. 2012.

[6] M. Gavalec, M. Gad, and K. Zimmermann, "Optimization problems under (max, min)linear equations and/or inequality constraints", Journal of Mathematical Sciences, Vol. 193, No.
5, pp. 645-658, Sep. 2013.

[7] R. Cimler, M. Gavalec, and K. Zimmermann, "An optimization problem on the image set of a (max, min) fuzzy operator", Fuzzy Sets and Systems, Vol. 341, pp. 113-122, 2018.

[8] G. Xiao, T. Zhu, Y. Chen, and X. Yang, "Linear searching method for solving approximate solution to system of max-min fuzzy relation equations with application in the instructional information resources allocation", IEEE Access, Vol. 7, pp. 65019-65028, 2019.

[9] A. El-sawy, and A. Tharwat, "Comparison of Particle SWARM Optimization, Genetic Algorithm and Max separable Technique for Machine Time Scheduling Problem", In: Proc. of The International Conf. on Mathematics and Engineering Physics, Vol. 5, No. 5, pp. 1-10, 2010.

[10] H. M. Rabie, I. A. El-Khodary, and A. A. Tharwat, "Particle swarm optimization algorithm for the continuous p-median location Problems", In: Proc. of 10th International Computer Engineering Conf.: Today Information Society What's Next?, ICENCO 2014, pp. 81-86, 2015.

[11] S.-M. Guo, P.-H. Hsu, C.-C. Yang, and J. S.-H. Tsai, "Constrained min-max optimization via the improved constraint-activated differential evolution with escape vectors", Expert Systems with Applications, Vol. 46, pp. 336-345, Mar. 2016.

[12] M. Khan, and J. E. Rice, "Hybrid GA synthesis of ternary reversible circuits using max-min algebra", Journal of Multiple-Valued Logic and Soft Computing, Vol. 32, No. 1-2, pp. 27-55, 2019.

[13] H. Garg, "A hybrid PSO-GA algorithm for constrained optimization problems", Applied Mathematics and Computation, Vol. 274, pp. 292-305, Feb. 2016.

[14] E. S. Barroso, E. Parente, and A. M. Cartaxo de Melo, "A hybrid PSO-GA algorithm for optimization of laminated composites", Structural and Multidisciplinary Optimization, Vol. 55, No. 6, pp. 2111-2130, Jun. 2017.

[15] M. Ahmad, W. Ali, H. Farooq, M. Jamil, M. Ali, and A. U. Rehman, "Solving the Problem of Economic Load Dispatch for a Small Scale Power System Using a Novel Hybrid PSO-GSA Algorithm", In: Proc. of 2018 International Symposium on Recent Advances in Electrical Engineering (RAEE), pp. 1-6, 2018.

[16] S. Yan, Q. Liu, J. Li, and L. Han, "Heterogeneous Acceleration of Hybrid PSOQN Algorithm for Neural Network Training", 
IEEE Access, Vol. 7, pp. 161499-161509, 2019.

[17] A. Ansari, I. S. Ahmad, A. A. Bakar, and M. R. Yaakub, "A Hybrid Metaheuristic Method in Training Artificial Neural Network for Bankruptcy Prediction”, IEEE Access, Vol. 8, pp. 176640-176650, Sep. 2020.

[18] B. A. Mitras, and D. A. Anwar, "Using hybrid PSO algorithm with modified conjugate gradient method for some image processing", In: Proc of AIP Conf. Proceedings, pp. 1-16, 2019.

[19] S.-H. Xu, J.-P. Liu, F.-H. Zhang, L. Wang, and L.-J. Sun, "A Combination of Genetic Algorithm and Particle Swarm Optimization for Vehicle Routing Problem with Time Windows", Sensors, Vol. 15, No. 9, pp. 21033-21053, Aug. 2015.

[20] N. Niazy, A. El-Sawy, and M. Gadallah, "A hybrid chicken swarm optimization with tabu search algorithm for solving capacitated vehicle routing problem", International Journal of Intelligent Engineering and Systems, Vol. 13, No. 4, pp. 237-247, 2020.

[21] N. Niazy, A. El-Sawy, and M. Gadallah, "Solving Capacitated Vehicle Routing Problem Using Chicken Swarm Optimization with Genetic Algorithm", International Journal of Intelligent Engineering and Systems, Vol. 13, No. 5, pp. 502-513, Oct. 2020.

[22] M. A. M. Gad, "Optimization Problems Under (max, min)-Linear Constraints and Some Related Topics", PhD thesis, Faculty of Mathematics and Physics, Charles University Prague, 2015.

[23] M. Kumar, M. Husain, N. Upreti, and D. Gupta, "Genetic Algorithm: Review and Application", International Journal of Information Technology and Knowledge Management, Vol. 2, No. 2, pp. 451-454, 2010.

[24] K. Deep, K. P. Singh, M. L. Kansal, and C. Mohan, "A real coded genetic algorithm for solving integer and mixed integer optimization problems", Applied Mathematics and Computation, Vol. 212, No. 2, pp. 505-518, Jun. 2009.

[25] K. Masuda, K. Kurihara, and E. Aiyoshi, "A penalty approach to handle inequality constraints in particle swarm optimization", In: Proc. of 2010 IEEE International Conf. on Systems, Man and Cybernetics, pp. 2520-2525 Oct. 2010.

[26] M. A. Arasomwan, and A. O. Adewumi, "On the performance of linear decreasing inertia weight particle swarm optimization for global optimization", The Scientific World Journal, Vol. 2013, pp.1-12, 2013.
[27] S. M. A. Salehizadeh, P. Yadmellat, and M. B. Menhaj, "Local optima avoidable particle swarm optimization," In: Proc. of 2009 IEEE Swarm Intelligence Symposium, SIS 2009 Proceedings, 2009, pp. 16-21.

[28] W. Chinnasri, "Adaptive Probability of Crossover and Mutation in Genetic Algorithm on University Course Timetabling Problem Adaptive Probability of Crossover and Mutation in Genetic Algorithm on University Course Timetabling Problem", In: Proc. of CSAE 2013 : 2013 IEEE International Conf. on Computer Science and Automation Engineering, No. March, pp. 725-728, 2015.

[29] M. Kuehn, T. Severin, J. Lippold, H. Salzwedel, and V. Nissen, "Adaptive Mutation Rate at Genetic Algorithms with Multi-Chromosome Representation in Multi-department Hospital Process Optimization", International Journal of Computer Applications, Vol. 182, pp. 41-52, Oct. 2018. 